\title{
BAIRE CLASS 1 SELECTORS FOR UPPER SEMICONTINUOUS SET-VALUED MAPS
}

\author{
V. V. SRIVATSA
}

\begin{abstract}
Let $T$ be a metric space and $X$ a Banach space. Let $F: T \rightarrow X$ be a set-valued map assuming arbitrary values and satisfying the upper semicontinuity condition: $\{t \in T: F(t) \cap C \neq \varnothing\}$ is closed for each weakly closed set $C$ in $X$. Then there is a sequence of norm-continuous functions converging pointwise (in the norm) to a selection for $F$. We prove a statement of similar precision and generality when $X$ is a metric space.
\end{abstract}

\section{INTRODUCTION}

Several articles have appeared in recent years on upper semicontinuous setvalued maps and, in particular, on the selection problem which we shall be mainly concerned with in this paper-notably the work of Jayne, Rogers, and their collaborators. For the sake of brevity, we shall confine ourselves to stating the recent results that led most directly to ours and a few remarks motivating our work. We urge the reader to consult the papers listed in the bibliography for earlier history and a more elaborate description of the problem (we would particularly recommend $[9,10,12])$.

In what follows $T$ will always be a metric space, $X$ a topological space (we shall be interested in the cases when $X$ is a normed linear space with its weak topology or a metric space), and $F: T \rightarrow X$ a set-valued map on $T$ into $\mathscr{P}(X)-\varnothing$. We postpone all definitions to $\S 1$.

The statements below are only meant to be indicative and some of them are stated in versions slightly weaker than in the original-to which we refer the reader.

Theorem A (Jayne-Rogers [9]). Let $X$ be metric and $F: T \rightarrow X$ be upper semicontinuous. Then $F$ has a Borel class 2 selection.

Theorem B (Jayne-Rogers [9], see also Hansell [5]). Let $X$ be metric. If $F: T \rightarrow$ $X$ is upper semicontinuous and $F(t)$ is complete for each $t$, then $F$ has a Borel class 1 selection.

Many interesting examples of upper semicontinuous maps arise as ones taking values in a Banach space, e.g., maximal monotone maps (of which the subdifferential is a special case), attainment maps, and metric projections (see [10,

Received by the editors September 11, 1990.

1980 Mathematics Subject Classification (1985 Revision). Primary 54C60, 54C65, 46B99.

Key words and phrases. Upper semicontinuous set-valued maps, Baire class 1 maps, selectors, weak and weak* topologies. 
$11,12])$. Here the problem becomes more subtle (and difficult) owing to the presence of more than one topology. Indeed, in this context, the upper semicontinuity is usually with respect to (the possibly nonmetrizable) weak and weak* topologies.

Theorem C (Jayne-Rogers [10]). Let $F$ be a weakly upper semicontinuous setvalued map on $T$ taking values in a fixed weakly compact subset of a Banach space $X$. Then $F$ has a norm Borel selector. When, in addition, $F$ assumes only weakly compact values, there is one of the second Borel class.

Theorem D (Hansel-Jayne-Talagrand [7]). Suppose $T$ is hereditarily Baire and $X$ is a Banach space. If $F$ is weakly upper semicontinuous and weakly compactvalued, then $F$ has a norm Baire class 1 selection.

Theorem E (Jayne-Rogers [11]). Let $X$ (respectively $X^{*}$ ) be a Banach space (respectively a dual) with the Radon-Nikodym property. Suppose $F$ is weakly (respectively weak*) compact-valued and weakly (resp. weak*) upper semicontinuous. Then $F$ has a Baire class 1 selection.

As the variety of hypotheses in the theorems above suggests, their proofs are based on different principles. The proof of Theorem A hinges on the Choquet 'boundary' of $F$ and a careful calculation of the topological nature of the graph of $F$. Hansell's proof of Theorem B on the other hand is on the classical Kuratowski-Ryll-Nardzewski [14] pattern in a nonseparable version. In Theorem $\mathrm{C}$, the authors invoke a theorem of Amir and Lindenstrauss to restrict attention to spaces of type $C_{0}(\Gamma)$, and special geometric properties of $C_{0}(\Gamma)$ enable them to carry over the arguments in [9]-a complex argument. Theorem $\mathrm{D}$, in a quite different spirit, is based on Baire category arguments through the use of a recent Namioka type theorem of Chistensen-Saint-Raymond [4, 17] stating that any minimal, compact-valued upper semicontinuous map is singlevalued on a dense $G_{\delta}$ set. Finally, the proof of Theorem E proceeds via the formulation of the interesting notion of a topology 'fragmentable by a metric.' The observation that the Radon-Nikodým property ensures that on any bounded subset the weak (or weak*) topology is 'fragmentable' by the norm allows the authors to obtain a selection on the Kuratowski-Ryll-Nardzewski principle.

Our work arose in an attempt to understand these theorems and see them in a single scheme and in the hope that thereby one could dispense with the technical hypotheses. In this article we shall present a complete solution in the case of maps taking values in a Banach space (actually any normed linear space) with its weak topology (which, with some qualification subsumes the metric case)-namely,

Theorem. Let $T$ be a metric space and $X$ a Banach space. Let $F: T \rightarrow X$ be a set-valued map assuming arbitrary values and satisfying the upper semicontinuity condition: $\{t \in T: F(t) \cap C \neq \varnothing\}$ is closed for each weakly closed set $C$ in $X$. Then there is a sequence of norm-continuous functions converging pointwise (in the norm) to a selection for $F$.

It turns out that arguments elementary in principle yield precise results in complete generality. A key ingredient is the proof of the special case (a statement of which we have been unable to find in the literature!): any weakly continuous (point-valued) map on a metric space into a Banach space is a pointwise 
(norm) limit of a sequence of norm-continuous functions. While such distinctions are misleading in the multi-valued case, notice that in the separable case the above easily follows from the fact that a weakly open set in a separable Banach space is a norm $F_{\sigma}$ (and was indeed established by Alexiewicz and Orlicz [1]). Even this corollary is perhaps not without interest. To see what this could mean we note that this yields a particularly transparent proof of the Orlicz-Pettis Theorem: every weakly unconditionally convergent series is norm unconditionally convergent.

A word on the class of the selectors. Easy (and well-known) examples show that our results are the best possible (and as far as we are aware, ours is the first instance when (even Borel) class 1 selectors have been obtained without any assumption on the values of $F$, in any situation). But quite apart from this, this seems to be the right level of precision to aim for. As Jayne and Rogers [12] have observed, the points of continuity of a selector have canonical properties in concrete cases. The underlying principle seems to be the following. Let us restrict attention to the important class of minimal (in the natural ordering through graph inclusion) upper semicontinuous, compact-valued maps (e.g., the maximal monotone maps). Assume further that the metric space $T$ is a Baire space. Let $F: T \rightarrow X$ be upper semicontinuous and compact-valued, and let $f$ be a (norm) Baire class 1 selection for $F$ obtained from the Theorem. By the relevant generalization of Baire's classical theorem the set of points of norm continuity of $f, C_{f}$, is a dense $G_{\delta}$. It is easily seen that the closure of $\left\{\langle x, f(x)\rangle: x \in C_{f}\right\}$ is the graph of an upper semicontinuous, compact-valued map, which must coincide with $F$ when $F$ is minimal. And so one has a proof of the theorem of Christensen-Saint-Raymond referred to earlier: any minimal, weakly upper semicontinuous, compact-valued $F$ is point-valued and norm upper semicontinuous on a dense $G_{\delta}$ set.

When $X$ is metric, the existence of Borel class 1 selectors follows immediately, via an appropriate embedding, from the Banach space case (since we make no assumptions on the values of $F$ ). Unfortunately, examples are easily constructed to show that one cannot in general obtain Baire class 1 selectors. However, even in the metric case our selectors do have the important property that they are continuous outside a meager set.

Some months after we had done our work we heard that Hansell had independently proved our Theorem in the cases when $X$ is a metric space or a separable Banach space (in both cases Theorems A-E guarantee the existence of Borel selectors). We have also learned that Jayne and Rogers have obtained some refinements of their results yielding, e.g., selectors of the respective Baire classes in Theorems A and B.

The paper is organized as follows. In $\S 1$ we fix the notation and do the preliminary work, and in $\S 2$ we establish the main results and put together several remarks and questions.

It is doubtful if the author would have embarked on this work had he not been visiting University College London during the academic year 1984-85. $\mathrm{He}$ is grateful to Professors Jayne and Rogers for arranging this visit, the Department of Mathematics for its hospitality, and the Science and Engineering Research Council, U.K., for generous financial assistance. Quite apart from his indebtedness to their work, the author wishes to acknowledge the discussions he had with Professors Jayne and Rogers, and for their comments and useful 
suggestions on a first draft. He shall particularly remember the kindness shown to him by Professor Rogers. Thanks are also due to Iwo Labuda, who was another visitor at University College, and to G. Debs for comments on the manuscript. We are also grateful to T. S. S. R. K. Rao for helpful conversations.

\section{Preliminaries}

In what follows $T$ will always stand for a metric space and $X$ for a topological space. The symbol $d$ will always stand for the distance function whatever the metric space under consideration. When $Y$ is a metric space and $y \in Y$, $B(y ; \varepsilon)$ will stand for the open ball of radius $\varepsilon$ centered at $y$. When $Y$ is a normed linear space we will simply write $B_{Y}$ for its unit ball. When $A$ is any set, $\bar{A}$ will denote its closure in the relevant topology.

A reference for most of the notions we use is Kuratowski [13], and we refer the reader to it for a general definition of the Borel and Baire classifications of sets and functions. Recall however that the sets of additive class 1 in $T$ are the $F_{\sigma}$ sets and the sets of multiplicative class 1 are the $G_{\delta}$ sets. Subsets of $T$ that are simultaneously $F_{\sigma}$ and $G_{\delta}$ are referred to as sets of ambiguous class 1 .

When $f$ is a function on $T$ into $X, f$ is said to be of Borel class 1 if the inverse image of every open set in $X$ is $F_{\sigma}$ in $T$. The function $f$ is Baire class 1 if it is a pointwise limit of continuous functions. The former of these two classes of functions is closed under uniform limits. The Baire class 1 functions are of special importance and have the following interesting property: the set of discontinuity points of any Baire class 1 function is a meager $F_{\sigma}$ in $T$ (for metrizable $X$ ). While we shall not meet any Borel functions of class bigger than one in what follows, we have referred to the class 2 functions in the introduction: a function $f: T \rightarrow X$ is Borel class 2 if $f^{-1}(V)(=\{t \in T: f(t) \in V\})$ is a countable union of $G_{\delta}$ sets for each open $V$ in $X$.

Every Baire class 1 function is always Borel class 1 (for metrizable range) and it is a classical theorem that when $X$ is the unit interval (more generally, a finite product of intervals), the converse holds. This is far from being the case in general. The selectors we construct will easily be seen to be Borel class 1. Furthermore, they arise as uniform limits of functions with a particularly simple structure and Proposition 1.3 below will enable us to conclude that they are actually Baire class 1 , when $X$ is a normed linear space. We now proceed to introduce this class of maps and put down a few of their properties-we shall meet them often in the rest of the paper.

We will find it convenient to deal with functions that may not be defined on the whole of $T$ (partial functions). We will continue to use the notation " $f: T \rightarrow X$ " even when $f$ is partial. However, a function $f: T \rightarrow X$ must always be taken to be defined on the whole of $T$ unless we explicitly use the epithet "partial."

Definition 1.1. Call a partial function $f: T \rightarrow X$ rudimentary if the domain of $f$ is the union of a discrete (in $T$ ) family of open sets on each of which $f$ is constant.

Notice that the definition of rudimentary depends on the underlying space $T$ even when $f$ may be defined only partially on it, since the domain of $f$ 
must be expressible as a union of a family (of open sets) discrete in $T$. Strictly speaking, for a partial function we should always say "rudimentary on $T$," but we have subsumed this by our notation $f: T \rightarrow X$ even when $f$ is partial, and shall just say "rudimentary" unless there is scope for confusion as in the next definition.

If $f: T \rightarrow X$ and $A \subseteq T$, then the abbreviation $f \mid A$ will denote the restriction of $f$ to $A$.

Definition 1.2. Call a function $f: T \rightarrow X$ rudimentary of class 1 if there is a sequence of pairwise disjoint ambiguous class 1 sets $A_{m}$ such that $\bigcup_{m \geq 1} A_{m}=$ $T$ and $f \mid A_{m}$ is rudimentary (on $A_{m}$ ) for each $m$.

The next two propositions are trivial.

Proposition 1.1. Suppose $\left\{B_{n}\right\}$ is a sequence of pairwise disjoint sets of ambiguous class 1 such that $\bigcup_{n \geq 1} B_{n}=T$ and $f_{n}: B_{n} \rightarrow X$ is rudimentary of class 1 for each $n$. Then $f: T \rightarrow X$, defined by

$$
f(t)=f_{n}(t) \quad \text { for } t \in B_{n},
$$

is rudimentary of class 1 .

Proposition 1.2. Let $\mathbf{V}$ be a discrete open cover for $T$. Suppose for each $V \in \mathbf{V}$ there is $f_{V}: V \rightarrow X$ rudimentary of class 1 . Then $f: T \rightarrow X$, defined by

$$
f(t)=f_{V}(t) \quad \text { for } t \in V,
$$

is rudimentary of class 1 .

We shall now observe that the classical arguments (see [13]) are easily modified to show that when $X$ is a normed linear space, any uniform limit of rudimentary class 1 functions is Baire class 1 (with respect to the norm topology on $X)$.

Lemma 1.1. Let $X$ be a normed linear space. Suppose $f$ and $g$ are rudimentary class 1 functions on $T$ into $X$ such that $\|f(t)-g(t)\| \leq \varepsilon$ for each $t \in T$. Assume further that $\left\{f_{n}\right\}$ is a sequence of continuous (in the norm) functions on $T$ into $X$ converging pointwise to $f$. Then there is a sequence of normcontinuous functions $g_{n} \rightarrow g$ pointwise such that for each $n,\left\|g_{n}(t)-f_{n}(t)\right\| \leq \varepsilon$ for all $t$.

Proof. Since $f$ and $g$ are rudimentary class 1 , it is easily seen that $g-f$ is rudimentary class 1 . Further, $g-f$ takes values in $\bar{B}(0 ; \varepsilon)$. Let $A_{m}$ be a sequence of pairwise disjoint ambiguous class 1 sets such that $\bigcup_{m \geq 1} A_{m}=T$ and such that $(g-f) \mid A_{m}$ is rudimentary for each $m$. For each $m \geq 1$ we may write $A_{m}=\bigcup_{n \geq 1} A_{m n}$, with $A_{m n}$ increasing and $A_{m n}$ closed for each $n$. Put $F_{n}=\bigcup_{m=1}^{n} A_{m n}$. Then each $F_{n}$ is closed and $\bigcup_{n \geq 1} F_{n}=T$.

Fix $n \geq 1$. Observe that $(g-f) \mid F_{n}$ is continuous on $F_{n}$. By the DugundjiBorsuk extension theorem (which applies since $X$ is a normed linear space [2]) $(g-f) \mid F_{n}$ has a continuous extension $h_{n}$ on the whole of $T$ into $\bar{B}(0 ; \varepsilon)$. It is easily checked that $h_{n} \rightarrow g-f$ pointwise. To complete the proof take $g_{n}=h_{n}+f_{n}$.

Proposition 1.3. Let $X$ be a normed linear space. If $f$ is the uniform limit of a sequence of rudimentary class 1 functions on $T$ into $X$, then $f$ is Baire class 1 (in the norm). 
Proof. Let $f_{n}: T \rightarrow X$ be a sequence of rudimentary class 1 functions converging uniformly to $f$ on $T$ into $X$. Without loss of generality we may assume that for each $n \geq 1,\left\|f_{n}(t)-f_{n+1}(t)\right\| \leq 1 / 2^{n}$ for all $t$. We shall now construct a double sequence of continuous functions $\left\{h_{n m}\right\}$ on $T$ into $X$ satisfying:

(i) for each $n \geq 1, h_{n m} \rightarrow f_{n}$ pointwise, and

(ii) for $n, m \geq 1,\left\|h_{n m}(t)-h_{n+1, m}(t)\right\|<1 / 2^{n}$.

The construction is by induction on $n$. For the base step, notice that the argument in Lemma 1.1 yields a sequence of continuous functions $\left\{h_{1 m}\right\}$ converging pointwise to $f_{1}$. Suppose $\left\{h_{n m}\right\}_{m \geq 1}$ has been constructed. Apply Lemma 1.1 to $f_{n}$ and $f_{n+1}$ (playing the role of $f$ and $g$ respectively) and the sequence $\left\{h_{n m}\right\}_{m \geq 1}$ with $\varepsilon=1 / 2^{n}$. Call the sequence given by the lemma $\left\{h_{n+1, m}\right\}_{m \geq 1}$. This does the job.

Finally, notice that for fixed $t \in T$, the inequality

$$
\begin{aligned}
\left\|h_{m m}(t)-f(t)\right\| \leq & \left\|h_{m m}(t)-h_{n m}(t)\right\|+\left\|h_{n m}(t)-f_{n}(t)\right\| \\
& +\left\|f_{n}(t)-f(t)\right\|
\end{aligned}
$$

in conjunction with (i), (ii), and the fact that $f_{n}(t) \rightarrow f(t)$ ensures that the diagonal sequence $\left\{h_{m m}\right\}$ converges pointwise to $f$.

What happens for general metric spaces $X$ ? Simple examples and the argument above (which is just the classical proof rewritten) suggest that a minimum requirement is that Tietze type extension theorems must hold for maps on $T$ into $X$. Hansell has claimed [6] that for such $X$ the Borel and Baire classifications do coincide for the large class of ' $\sigma$-discrete mappings.' All the maps we construct are easily checked to be $\sigma$-discrete. Why then have we taken the trouble to prove Proposition 1.3? Unfortunately, the proof of [6, Theorem 6] is not correct (and does not seem rectifiable) - since all the previous authors in the subject have invariably invoked Hansell, this must be a small gap in their proofs. ${ }^{1}$ However, as we shall observe in the next section, standard embedding arguments ensure that for our purposes Proposition 1.3 suffices.

When $f: T \rightarrow X$ and $X$ is a normed linear space, $f$ is weakly continuous if it is continuous with respect to the weak topology. Similarly norm-continuous, weakly Borel, etc., have the obvious meanings.

By a set-valued map (or multifunction) $F: T \rightarrow X$ we mean a function on $T$ into $\mathscr{P}(X)-\{\varnothing\}$. The graph of $F$ (notation: $\operatorname{Gr}(F))$ is the set $\{(t, x)$ : $x \in F(t)\}$. For $A \subseteq T, F(A)$ will denote $\{x \in X: x \in F(t)$ for some $t \in A\}$. The multifunction $F: T \rightarrow X$ is called upper semicontinuous (u.s.c.) if for each closed $C$ in $X$, the set $F^{-1}(C)=\{t \in T: F(t) \cap C \neq \varnothing\}$ is closed in $T$. These are natural objects. One way in which they pervade the literature is as set-valued 'continuous' extensions of continuous maps, when there is no continuous extension-the closure of the graph of the $\sin \frac{1}{x}$ function on $[0,1]$ being a prototype (see Labuda [15] for an elaboration on this theme). For examples in function spaces see $[10,11,12]$. We shall come across a couple of nonstandard examples later in the article. When $X$ is a normed linear space equipped with the weak topology, we shall call such an $F$ weakly u.s.c.; and when $X$ is equipped with the norm topology, norm u.s.c.

\footnotetext{
${ }^{1}$ The referee has pointed out that all the applications of the results of [6] are valid for an absolute
} extensor for metric spaces (in particular, a normed linear space). See [19, pp. 389-390]. 
There is a strong connection between upper semicontinuity and compactness. When $X$ is compact and $F$ is closed valued (i.e., $F(t)$ is closed for each $t$ ), $F$ is u.s.c. if and only if the graph of $F$ is closed in $T \times X$. The following, though simple, is the key fact about upper semicontinuity, and appears in some form or the other in every paper on the subject.

1.4. Let $F: T \rightarrow X$ be upper semicontinuous. Suppose a sequence $\left\{t_{n}\right\}_{n \geq 1}$ in $T$ converges to $t \in T$, and $x_{n} \in F\left(t_{n}\right)$. Then either $x_{n} \in F(t)$ for infinitely many $n$, or $\left\{x_{n}\right\}$ has a strict accumulation point $x \in F(t)$.

\section{THE MAIN RESUlTS}

For clarity and ease of exposition we shall split up the proof of our Theorem into several steps, essentially by proving special cases first. Our first step will be to present one ingredient in the proof by proving the point-valued case of our Theorem.

Theorem 2.1. Let $T$ be a metric space, and $X$ a normed linear space. Suppose $f: T \rightarrow X$ is a weakly continuous point-valued function. Then $f$ is a pointwise (norm) limit of a sequence of norm-continuous functions.

Proof. We will construct a sequence of rudimentary class 1 functions converging uniformly to $f$.

Since $T$ is metric there is a $\sigma$-discrete base for the topology on $T$. Therefore, fix a $\sigma$-discrete base $\mathbf{U}=\bigcup_{n \geq 1} \mathbf{U}_{n}$, where each $\mathbf{U}_{n}$ is a discrete family of open sets. For each $U \in \mathbf{U}$ choose and fix a $t_{U} \in U$ and put $x_{U}=f\left(t_{U}\right)$.

Define, for $n \geq 1$, the partial function $f_{n}: T \rightarrow X$ with domain $\bigcup\{V: V \in$ $\left.\mathbf{U}_{n}\right\}$ by

$$
f_{n}(t)=x_{U} \quad \text { if } t \in U, U \in \mathbf{U}_{n} .
$$

Since $\mathbf{U}_{n}$ is a discrete family, the definition above is unambiguous. Plainly, for each $n \geq 1$, the partial function $f_{n}$ is rudimentary. Furthermore, since $\mathbf{U}$ is a base for the topology on $T$, for each $t \in T$ and $n \geq 1$ there is an $m \geq 1$ such that $f_{m}(t)$ is defined and $f_{m}(t) \in f\left(B\left(t ; \frac{1}{n}\right)\right)$. Since $f$ is weakly continuous, it follows that, for each $t \in T$, there is a sequence $m_{1}, m_{2}, \ldots$ such that

$$
\begin{gathered}
f_{m_{k}}(t) \text { is defined for each } k \text {, and } \\
\qquad f_{m_{k}}(t) \stackrel{\mathrm{w}}{\longrightarrow} f(t) .
\end{gathered}
$$

By the Hahn-Banach theorem, there must therefore be a subsequence of the sequence of all finite rational linear combinations of the sequence $f_{n}(t)$ converging in the norm to $f(t)$.

Consider now the sequence of functions $\left\{g_{m}\right\}_{m \geq 1}$ obtained by taking all finite rational linear combinations of the functions $\left\{\bar{f}_{n}\right\}_{n \geq 1}$. It is easily checked that each $g_{m}$ is a rudimentary partial function on $T$. Furthermore, from what we have seen above we know that for each $t \in T$ and $\varepsilon>0$, there is an $m \geq 1$ such that

$$
g_{m}(t) \text { is defined and }\left\|f(t)-g_{m}(t)\right\| \leq \varepsilon .
$$

Fix $l \geq 1$ and put for $m \geq 1$,

$$
A_{m}^{l}=\left\{t \in T: g_{m}(t) \text { is defined and }\left\|f(t)-g_{m}(t)\right\| \leq 1 / l\right\} .
$$

In view of $(*)$ above, $\bigcup_{m \geq 1} A_{m}^{l}=T$. 
Let $\mathbf{V}$ be a discrete family of open sets such that $U \mathbf{V}=\operatorname{domain}\left(g_{m}\right)$ and such that $g_{m}$ takes the constant value $g_{m}^{V}$ on $V \in \mathbf{V}$. Then

$$
A_{m}^{l}=\bigcup\left\{V \cap\left\{t: f(t) \in \bar{B}\left(g_{m}^{V} ; 1 / l\right)\right\}: V \in \mathbf{V}\right\} .
$$

As $\mathbf{V}$ is discrete and $f$ is weakly continuous, $A_{m}^{l}$ is the intersection of a closed set and an open set, and so of ambiguous class 1 .

Define $h_{l}: T \rightarrow X$ by

$$
h_{l}(t)=g_{m}(t) \quad \text { if } t \in A_{m}^{l}-\bigcup_{k<m} A_{k}^{l} .
$$

For each $l \geq 1, h_{l}$ is rudimentary of class 1 , and plainly $h_{l} \rightarrow f$ uniformly in the norm. By Proposition 1.3, $f$ is Baire class 1 in the norm.

It is interesting to observe that the above yields a particularly transparent proof of the well-known Orlicz-Pettis theorem.

Corollary 2.1 (Orlicz-Pettis). Let $X$ be a Banach space. Every weakly unconditionally convergent series in $X$ converges unconditionally in the norm. Equivalently, every weakly subseries convergent series in $X$ is subseries convergent in the norm.

Proof. The equivalence is easy and well known. We will prove the subseries version. Let $\left\{x_{n}\right\}$ be a sequence in $X$ and assume by hypothesis that for every sequence $n_{1}<n_{2}<\cdots$ of integers, w- $\sum_{i=1}^{\infty} x_{n_{i}}$ exists. We now identify each such sequence $n_{1}<n_{2}<\cdots$ with the element of the Cantor space such that $\alpha(k)=1$ iff $k=n_{i}$ for some $i$. Consider now the map $F: 2^{\omega} \rightarrow X$ defined by

$$
F(\alpha)=\mathrm{w}-\sum_{i \geq 1} x_{n_{i}},
$$

where $n_{1}<n_{2}<\cdots$ is an enumeration (may be finite) in increasing order of $\{k: \alpha(k)=1\}$.

By hypothesis, $F$ is weakly continuous on $2^{\omega}$ into $X$. By Theorem 2.1, therefore, $F$ is a pointwise limit of norm continuous functions, and so (since $2^{\omega}$ is a complete metric space) $F$ has at least one point of norm continuity, $\alpha_{0}$. Let $m_{1}<m_{2}<\cdots$ be an enumeration of $\left\{k: \alpha_{0}(k)=1\right\}$. Assume to begin with that this sequence is infinite. Observe that since $F$ is norm-continuous at $\alpha_{0}$,

$$
\left\|\mathrm{w}-\sum_{i \geq k} x_{m_{i}}\right\| \rightarrow 0 \text { as } k \rightarrow \infty .
$$

Fix now any sequence $n_{1}<n_{2}<\cdots$. We have to show that $\sum_{i \geq 1} x_{n_{i}}$ exists. Let $\alpha_{k}$ be the element of $2^{\omega}$ which is 1 precisely on the set $\left\{m_{1}, \ldots, m_{k}\right\} \cup$ $\left\{n_{k+1}, n_{k+2}, \ldots\right\}$. Then $\alpha_{k} \rightarrow \alpha_{0}$, and so, by the norm continuity of $F$ at $\alpha_{0}$,

$$
\left\|\sum_{i=1}^{k} x_{m_{i}}+\mathrm{w}-\sum_{i \geq k+1} x_{n_{i}}-\mathrm{w}-\sum_{i \geq 1} x_{m_{i}}\right\| \rightarrow 0 \quad \text { as } k \rightarrow \infty .
$$


So

$$
\left\|\mathrm{w}-\sum_{i \geq k+1} x_{n_{i}}-\mathrm{w}-\sum_{i \geq k+1} x_{m_{i}}\right\| \rightarrow 0 \text { as } k \rightarrow \infty .
$$

By $(*)$, therefore,

$$
\left\|\mathrm{w}-\sum_{i \geq k+1} x_{n_{i}}\right\| \rightarrow 0 \quad \text { as } k \rightarrow \infty .
$$

It follows that the sequence $\sum_{i=1}^{k} x_{n_{i}}$ is Cauchy in the norm and this completes the proof when $\left\{k: \alpha_{0}(k)=1\right\}$ is infinite. The other case is similar.

One reason for writing out Theorem 2.1 separately is that it brings out clearly the central role of the metric nature of the domain and the Hahn-Banach theorem-that is one ingredient in the proof of our Theorem. The rest of the proof is essentially a careful combination of the above with $\S 1.4$. The next lemma is basically the proof of the Theorem for closed-valued $F$.

Lemma 2.1. Suppose $X$ is a normed linear space and $F: T \rightarrow X$ a weakly u.s.c. set-valued map. Then there is a sequence $\left\{h_{n}\right\}_{n \geq 1}$ of rudimentary class 1 mappings on $T$ into $X$ satisfying

(i) $\left\|h_{n}(t)-h_{n+1}(t)\right\| \leq 1 / 2^{n} \quad \forall t$, and

(ii) $\bar{B}\left(h_{n}(t) ; 1 / 2^{n}\right) \cap \bar{F}(t) \neq \varnothing$.

Proof. As in the last theorem, by fixing a $\sigma$-discrete base $\mathbf{U}=\bigcup_{n \geq 1} \mathbf{U}_{n}$ for $T$, with each $\mathbf{U}_{n}$ discrete, we can get a sequence of rudimentary partial functions $\left\{f_{n}(t)\right\}$ such that (now), if again we pick (and fix) $t_{V} \in V$ and $x_{V} \in F\left(t_{V}\right)$ for each $V \in \mathbf{U}_{n}$, we have, $f_{n}(t) \in F\left(t_{V}\right)$ for $t \in V$ (via the definition $f_{n}(t)=x_{V}$ for $t \in V)$.

Again, for each $k \geq 1$, and every $t$, there is an $m \geq 1$ such that

$$
f_{m}(t) \text { is defined and } f_{m}(t) \in F(B(t ; 1 / k)) \text {. }
$$

Observe now that, as $F$ is u.s.c., $§ 1.4$ applies to yield: either $f_{m}(t) \in F(t)$ for infinitely many $m$ or there is a (strict) weak cluster point of $\left\{f_{m}(t)\right\}$ which belongs to $F(t)$. In either case, there is a subsequence of the sequence of finite rational linear combinations of $\left\{f_{m}(t)\right\}$ converging in the norm to a point in $F(t)$.

Let $\left\{g_{m}(t)\right\}_{m \geq 1}$ stand for the sequence (enumerated in some order) of rudimentary partial functions obtained by taking all finite rational linear combinations of the functions $\left\{f_{n}(t)\right\}_{n \geq 1}$. Then, by the above, for each $t \in T$ and $n \geq 1$, there is an $m$ such that $g_{m}(t)$ is defined and $\bar{B}\left(g_{m}(t) ; 1 / 2^{n}\right) \cap F(t) \neq \varnothing$. Let, for fixed $m \geq 1$,

$$
A_{m}=\left\{t \in T: g_{m}(t) \text { is defined and } \bar{B}\left(g_{m}(t) ; 1\right) \cap F(t) \neq \varnothing\right\} \text {. }
$$

Then $\bigcup_{m \geq 1} A_{m}=T$. Let $\mathbf{V}$ be a discrete family of open sets such that $\bigcup_{V \in \mathbf{V}} V=$ domain of $g_{m}$, and such that $g_{m}$ assumes the constant value $c_{V}$ on $V$. Then

$$
A_{m}=\bigcup_{V \in \mathbf{V}}\left(V \cap\left\{t \in T: \bar{B}\left(c_{V} ; 1\right) \cap F(t) \neq \varnothing\right\}\right)
$$


As $F$ is u.s.c, and $\mathbf{V}$ is discrete, it follows that $A_{m}$ is the intersection of an open set and a closed set, and so of ambiguous class 1 .

Define $h_{1}: T \rightarrow X$ by

$$
h_{1}(t)=g_{m}(t) \quad \text { if } t \in A_{m}-\bigcup_{k<m} A_{k} .
$$

Plainly, $h_{1}$ satisfies the required properties. Suppose $h_{n}$ has been defined to satisfy

$$
\bar{B}\left(h_{n}(t) ; 1 / 2^{n}\right) \cap F(t) \neq \varnothing .
$$

We will now define $h_{n+1}$ satisfying

(i) $d\left(h_{n}(t), h_{n+1}(t)\right) \leq 1 / 2^{n}$, and

(ii) $\bar{B}\left(h_{n+1}(t) ; 1 / 2^{n+1}\right) \cap F(t) \neq \varnothing$.

As $h_{n}$ is rudimentary of class 1 (by the induction hypothesis), there is a sequence $\left\{B_{m}\right\}$ of ambiguous class 1 sets such that $h_{n} \mid B_{n}$ is rudimentary (on $B_{m}$ ) for each $m \geq 1$. By Proposition 1.1, it is enough to construct $h_{n+1}$ on each $B_{m}$ separately, so we may assume without loss that $h_{n}$ is rudimentary. Similarly, by Proposition 1.2 we may assume $h_{n}$ is constant. Let then the constant value of $h_{n}$ be $c$. By the induction hypothesis, we know that $\bar{B}\left(c ; 1 / 2^{n}\right) \cap F(t) \neq \varnothing$ for all $t \in T$. Consider the set-valued mapping $F^{\prime}: T \rightarrow \bar{B}\left(c ; 1 / 2^{n}\right)$ defined by

$$
F^{\prime}(t)=F(t) \cap \bar{B}\left(c ; 1 / 2^{n}\right) .
$$

Then, since $\bar{B}\left(c ; 1 / 2^{n}\right)$ is weakly closed, $F^{\prime}$ is weakly u.s.c., and furthermore $F^{\prime}$ is nonempty valued. Repeat the procedure in the base step (the construction of $h_{1}$ ) to get (now) $h_{n+1}: T \rightarrow \bar{B}\left(c ; 1 / 2^{n}\right)$ rudimentary of class 1 so that $\bar{B}\left(h_{n+1}(t) ; 1 / 2^{n+1}\right) \cap F(t) \neq \varnothing$. This completes the induction.

Remark 2.1. For Banach spaces $X$, the above suffices to yield the selection theorem for closed-valued $F$. For, let $F: T \rightarrow X$ be weakly u.s.c. and weakly closed-valued. Get a sequence $\left\{h_{n}\right\}$ as in the lemma. Then $\left\{h_{n}\right\}$ is uniformly Cauchy in the norm and so (norm) $\lim h_{n}=h$ exists. By Proposition $1.3, h$ is Baire class 1 to the norm, and since $F$ is closed-valued, $h(t) \in F(t)$ for each $t$.

In view of the proof of the lemma, there is the appearance of sleight of hand to the next lemma. But it is only an intermediate selection theorem. It is of some surprise though that an 'approximate selection' of such precision exists.

Lemma 2.2. Let $X$ be a normed linear space and $F: T \rightarrow X$ weakly u.s.c. Suppose $h: T \rightarrow X$ is rudimentary of class 1 and $(\forall t) \quad \bar{B}(h(t) ; \varepsilon) \cap F(t) \neq$ $\varnothing, \varepsilon>0$. Then there is $h^{\prime}: T \rightarrow X, h^{\prime}$ rudimentary of class 1 , such that $\left\|h(t)-h^{\prime}(t)\right\| \leq \varepsilon$ for all $t$, and $h^{\prime}(t) \in F(B(t ; \varepsilon))$ for every $t$.

Proof. Once again, as in the previous lemma, we may apply Propositions 1.1 and 1.2 to assume without loss of generality that $h$ is constant on $T$. Let this constant value be $c$. As before consider $F^{\prime}: T \rightarrow \bar{B}(c ; \varepsilon)$ defined by

$$
F^{\prime}(t)=F(t) \cap \bar{B}(c ; \varepsilon), \quad t \in T .
$$

Again, $F^{\prime}$ is weakly u.s.c. and nonempty valued. Fix a $\sigma$-discrete refinement $\mathbf{V}$ of the cover for $T$ consisting of the open sets of diameter less than $\varepsilon$. As 
before we can get a sequence of rudimentary partial functions $\left\{f_{n}\right\}_{n \geq 1}$ such that (now), for each $t \in T$, there is $n$ satisfying

$$
f_{n}(t) \text { is defined and } f_{n}(t) \in F^{\prime}(B(t ; \varepsilon)) .
$$

Each $f_{n}$ has open domain. Clearly the oft used standard disjointification procedure yields the required $h^{\prime}$.

We now come to the last step of our proof and establish our main theorem. The role of the previous lemma now becomes clear. The argument is perhaps not entirely obvious.

Theorem 2.2. Let $T$ be a metric space and $X$ a normed linear space. Suppose $F: T \rightarrow X$ is a weakly upper semicontinuous set-valued mapping taking arbitrary values. Then there is a sequence of norm continuous functions $f_{n}$ on $T$ into $X$ converging pointwise (in the norm) to $f: T \rightarrow X$ such that $f(t) \in F(t)$ for each $t \in T$. In particular, $F$ has a Borel class 1 selection whose set of (norm) discontinuity points is a meager $F_{\sigma}$.

Proof. By Lemma 2.1 there is a sequence of rudimentary class 1 functions $\left\{h_{n}\right\}$ satisfying:

(i) $d\left(h_{n}(t), h_{n+1}(t)\right) \leq 1 / 2^{n}$, and

(ii) $\bar{B}\left(h_{n}(t) ; 1 / 2^{n}\right) \cap \bar{F}(t) \neq \varnothing$, for each $t$.

By Lemma 2.2 there is, for each $n$, a rudimentary class 1 function $h_{n}^{\prime}$ such that

(iii) $d\left(h_{n}^{\prime}(t), h_{n}(t)\right) \leq 1 / 2^{n}$, and

(iv) $h_{n}^{\prime}(t) \in F\left(B\left(t ; 1 / 2^{n}\right)\right)$ for each $t$.

Let

$$
A_{1}=\left\{t \in T: h_{1}^{\prime}(t) \in F(t)\right\} \text {. }
$$

As $h_{1}^{\prime}$ is rudimentary class $1, A_{1}$ is, arguing as before, ambiguous of class 1 . More generally, put

$$
A_{n}=\left\{t \in T-\bigcup_{k<n} A_{k}: h_{n}^{\prime}(t) \in F(t)\right\}, \quad n \geq 2 .
$$

Then, by induction, each $A_{n}$ is ambiguous class 1 . Define now the sequence $\left\{f_{n}\right\}_{n \geq 1}$ of rudimentary class 1 functions by the recursion: $f_{1}(t)=h_{1}^{\prime}(t)$,

$$
f_{n+1}(t)=\left\{\begin{array}{lc}
h_{1}^{\prime}(t) & \text { for } t \in A_{1} \\
\vdots & \vdots \\
h_{n}^{\prime}(t) & \text { for } t \in A_{n} \\
h_{n+1}^{\prime}(t) & \text { otherwise }
\end{array}\right.
$$

Each $f_{n}$ is clearly rudimentary of class 1 . Furthermore, for $t \notin \bigcup_{n \geq 1} A_{n}$,

$$
f_{m}(t)=h_{m}^{\prime}(t) \notin F(t) \text { for every } m
$$

and, for $t \in A_{n}$,

$$
f_{n}(t)=f_{m+1}(t)=h_{n}^{\prime}(t) \text { for every } m \geq n .
$$

Plainly $f_{n}$ is uniformly Cauchy on $T$. Let us now observe that (norm) $\lim f_{n}(t)$ exists and belongs to $F(t)$ for every $t$. For $t \in A_{m}, f_{n}(t)$ is ultimately (in 
n) $h_{m}^{\prime}(t)$ and so $\lim f_{n}(t)$ exists and $\lim f_{n}(t)=h_{m}^{\prime}(t) \in F(t)$, by definition of $A_{m}$.

For $t \notin \bigcup_{m \geq 1} A_{m}, f_{n}(t)=h_{n}^{\prime}(t) \notin F(t) \quad(\forall n)$. By (iv) and $\S 1.4, f_{n}(t)$ has a weak cluster point, say $x \in F(t)$. But $f_{n}(t)$ is norm-Cauchy. It follows that $f_{n}(t) \rightarrow x$ (in the norm). Thus, for every $t, \lim f_{n}(t)$ exists and belongs to $F(t)$.

Finally, $f=\lim f_{n}$, being a uniform limit of rudimentary class 1 functions, is by Proposition 1.3, Baire class 1 (in the norm).

Remark 2.2. We must stress that as far as Borel class 1 selectors are concerned the theorem for normed linear spaces is not really more general than the corresponding statement for Banach spaces. This is because if $F: T \rightarrow X$ is as in the Theorem, embed $X$ in its second dual $X^{* *}$, and notice that $F$ continues to have its properties viewed as a map taking values in the Banach space $X^{* *}$ (it is important here that we prove a theorem making no assumptions on the values of $F$ ). So if the Theorem is proved for Banach spaces and there is a Borel class 1 selector for $F$, taking values in $X^{* *}$, this selector must take values in $X$, and does the job. However, this argument cannot be used for Baire class 1 selectors.

Let us now consider the case when $X$ is any metric space. It is not hard to see that the arguments we have presented in this section are actually simpler in this case without the complication of a nonmetrizable weak topology to deal with. The only point at which the argument breaks down is in our use of Proposition 1.3. That said, we will now put down the following as a corollary to the Theorem.

Corollary 2.2. Let $T$ and $X$ be metric spaces and $F: T \rightarrow X$ an upper semicontinuous set-valued mapping taking arbitrary values. Then $F$ has a Borel class 1 selection whose set of discontinuity points is a meager $F_{\sigma}$.

Proof. Since both the hypothesis and the conclusion depend only on the topology of $X$, we may assume without loss that $X$ is equipped with a metric $d$ such that $d$-diameter $(X) \leq 1$. It is clear then that the mapping $x \rightarrow(y \rightarrow d(x, y))$ is an isometric embedding of $X$ into the Banach space $B_{R}(X)$ of bounded, continuous real-valued functions on $X$ with the sup norm. Thus Theorem 2.2 (indeed in the much weaker form with no reference to the weak topology) applies to the multifunction $F$ viewed as one taking values in $B_{R}(X)$ to yield a Borel class 1 selection $f$ such that $f$ is continuous outside a meager $F_{\sigma}$. It is clear that $f$ must take values in $X$ (being a selection for $F$ ), and hence the result. Once again, as in the last remark, in using such an embedding argument it is important that we have proved the Theorem for Banach spaces making no assumption on the values of $F$.

A slightly harder embedding argument (for which the author is grateful to C. A. Rogers) yields Baire class 1 selectors in a situation already considered by several authors (see, e.g., [7]).

Corollary 2.3. Let $T$ be a metric space, $X$ a metrizable absolute retract, and $F: T \rightarrow X$ an upper semicontinuous set-valued mapping taking arbitrary values. Then $F$ has a Baire class 1 selection.

Proof. By a result in [2], $X$ can be embedded isometrically as a closed, linearly independent subset in a normed linear space $E$. Since $X$ is an absolute retract, 
$X$ is a retract of $E$, and so there is a continuous $g: E \rightarrow X$ such that $g \mid X$ is identity. Let $f_{n}: T \rightarrow E$ be a sequence of continuous functions converging pointwise to a selection $f$ for $F$ viewed as a mapping taking values in $E$, obtained from the Theorem. Notice that $g \circ f_{n} \rightarrow g \circ f$ pointwise and $g \circ f=f$. This does the job.

Remark 2.3. In an earlier version we only had the above for completely metrizable retracts, but C. A. Rogers pointed out the result in [2] to us. We have stuck to the embedding into $B_{R}(X)$ in Corollary 2.2 because it is simpler. It was also pointed out to us by C. A. Rogers that the condition 'metrizable absolute retract' is equivalent to the 'metrizable spaces with the extension property' considered by him and his collaborators (see [3, p. 21]).

Remark 2.4. The arguments in this remark are related to the Jayne-Rogers observations [12] on points of continuity of the selectors in various cases. Suppose the hypotheses of Theorem 2.2 or Corollary 2.2 are in force. Assume further that $F: T \rightarrow X$ is compact-valued and $T$ is a Baire space. Let $f: T \rightarrow X$ be a selection for $F$ of the kind guaranteed by the above results. Then $C_{f}=\{t \in T: f$ is continuous at $t\}$ is a dense $G_{\delta}$. Consider the multifunction $G: T \rightarrow X$ whose graph is the closure in $T \times X$ of $\left\{(t, f(t)): t \in C_{f}\right\}$. It is not hard to see (using the upper semicontinuity of $F$ and the compactness of the values of $F$ ) that $G$ is u.s.c., $G(t) \subseteq F(t)$ for each $t$, and $G(t)=\{f(t)\}$ for $t \in C_{f}$. Since $C_{f}$ is dense in $T$, it follows that $G$ does not properly contain the graph of any other u.s.c., compact-valued mapping. In other words, $G$ is minimal. In particular if $F$ is minimal, $F$ must coincide with $G$ and so $F$ must be singlevalued and continuous (in the normed linear space case, norm-continuous) on $C_{f}$. This is the theorem of Christensen-Saint-Raymond for metric domains. (Actually Christensen [4] showed that this is valid when the domain satisfies a certain game-theoretic condition and Saint-Raymond [17] showed that Christensen's result remains valid under a weaker (game-theoretic) condition which he showed was equivalent (in the category for metric spaces) to saying that $T$ is a Baire space. For metric domains, ours is a more precise result.)

Remark 2.5. Notice that the above argument also shows that any compact valued, u.s.c. $F: T \rightarrow X$ always contains a minimal such $G$ without the usual brute recourse to Hausdorff maximality arguments. This must be tempered by the remark that we have made heavy use of the axiom of choice. This is unavoidable since we make no assumptions on the values of $F$. Observe however that when $T$ and $X$ are both separable metrics we only use the countable axiom of choice. One cannot do better.

Remark 2.6. An interesting (and unusual) aspect of this selection theorem is that any distinction between separable and nonseparable domains is artificial and misleading. Indeed we would have found it much harder to obtain a precise version if we had concentrated (as we initially did) solely on the separable case, where $\sigma$-discrete covering arguments are not quite so natural. Another curious feature (related to Remark 2.5), at least for a descriptive set-theorist, is what appears to be the essentially noneffective nature of the Theorem.

Remark 2.7. It is natural (and important) to ask about possible extensions of our Theorem to nonmetric domains, and particularly to classes of compact Hausdorff spaces including the weakly compact sets. To fix ideas let $T$ be weakly 
compact. It is doubtful if even the point-valued version (Theorem 2.1) holds in complete generality. ${ }^{2}$ Recall, however, Namioka's theorem that on any weakly compact set, the weak and norm topologies coincide on a dense $G_{\delta}$, which must therefore be metrizable. Thus almost everywhere versions (in the sense of Baire category) are true when $T$ is weakly compact. In particular, Remark 2.4 remains valid when $T$ is weakly compact (and has indeed been established for any compact Hausdorff domain by Christensen). Moral: selection theorem + single-valued Namioka $\Rightarrow$ multivalued Namioka.

Remark 2.8. We have been silent so far on the weak* topology. Jayne and Rogers have observed [11] that the validity of Theorem E actually characterises the RNP in dual spaces with the weak* topology. (Reason: almost everywhere Fréchet differentiability of all continuous, convex functions implies RNP.) While this is almost a complete solution (for the weak* topology), a few questions remain. The most obvious one is whether the assumption on the compactness of the values can be dropped when $X^{*}$ has RNP. There is some hope here because Jayne and Rogers do not seem to use the full power of the upper semicontinuity. Indeed, their proof, with one obvious modification, works equally well when $F$ is lower semicontinuous (we shall resist the temptation of supplying a proof here).

In another direction, one might ask if weak* class 1 selectors (as opposed to norm Borel selectors) always exist in the $\left(X^{*}\right.$, weak*) case. Jayne and Rogers [11] have already considered this question and have obtained positive answers in some cases (including $L_{\infty}$ for example). In fact, Theorem 10(b) of [11] proves the weak* version of Theorem 2.2 in the case when $F$ has weak* closed values, and Hansell [19] proves it in the separable case.

Remark 2.9. The above also raises the question of whether even the point-valued version (namely the analogue of Theorem 2.1) characterizes the RNP in dual spaces. This is indeed true when $X$ is separable. The unit ball of $X^{*}, B_{X^{*}}$, is then metrizable. But then, if Theorem 2.1 holds (in the weak* version), it must apply to the metrizable domain $B_{X^{*}}$, and so the identity map on $B_{X^{*}}$ must be a pointwise (norm) limit of norm-continuous functions. It is easily checked that this forces $B_{X^{*}}$ to be separable in the norm, and so $X^{*}$ is separable. But then $X^{*}$ must have RNP.

Recall now Stegall's characterization of RNP in $X^{*}$ : every separable subspace $Y$ of $X$ has a separable dual. This raises the following question: Is there a weak*-weak* continuous (or even Borel) selection for the set-valued map $F: B_{Y^{*}} \rightarrow X^{*}$ defined by

$$
F(t)=\left\{y \in B_{X^{*}}: y \text { extends } t\right\} ?
$$

Notice that $F$ is a compact, convex-valued (in a very strong sense) upper semicontinuous set-valued map.

However (and we owe the following remark to T. S. S. R. K. Rao), recall that a Grothendieck space is a space $X$ such that in $X^{*}$, weak and weak* sequential convergence coincide. An obvious argument now shows that Theorem 2.1 holds (in the weak* version) for such an $X^{*}$. However there are Grothendieck spaces

\footnotetext{
${ }^{2}$ The referee has pointed out that if $T \subseteq X$ is weakly compact, then the identity $1_{T}$ is norm Baire class 1 iff $T$ is separable. So Theorem 2.1 does not hold in general.
} 
$X$ for which $X^{*}$ fails RNP. So we modify our question to: What are the spaces for which the analogue of Theorem 2.1 (with weak* replacing weak) hold.

Remark 2.10. Theorems 2.1 and 2.2 raise the following question on the structure of upper semicontinuous maps: Can every compact-valued, weakly u.s.c. $F: T \rightarrow X$ be expressed as a 'limit' of a sequence of norm u.s.c. ones?

Remark 2.11. Another natural structural question to ask about u.s.c. maps, particularly keeping in mind Remark 2.4 and the arguments in [7] is: Does every weakly u.s.c., compact-valued $F$ on a metric space $T$ into a Banach space $X$ have a weakly u.s.c. compact-valued extension $F: \mathbf{T} \rightarrow X$, where $\mathrm{T}$ is some completely metrizable space containing $T$ ? It is not hard to see that this is true whenever the range of $F$ is compact. Unfortunately, this is not always the case. The space $c_{0}$ (for $X$ ) provides an example-we shall outline a proof. To begin with, it can be argued that the identity map on $Q$ into $Q(Q \equiv$ rationals) has no set-valued u.s.c. extension (taking values in $Q$ ) to any completely metrizable domain. Now the unit ball of $c_{0}, B_{c_{0}}$, is (in the weak topology) separable metric, and being an injective continuous image of the norm topology, absolute Borel. However it is not a Baire space and therefore not completely metrizable. By a theorem of Hurewicz [8], $\left(B_{c_{0}}\right.$, weak) must contain a copy of $Q$ as a $G_{\delta}$.

It would be interesting to know when such extensions are possible.

\section{REFERENCES}

1. A. Alexiewicz and W. Orlicz, Sur la continuite et la classification de Baire des fonctions abstraites, Fund. Math. 35 (1948), 105-126.

2. C. Bessaga and A. Pelczynski, Selected topics in infinite dimensional topology, PWN, Warsaw, 1975.

3. K. Borsuk, Shape theory, Monogr. Mat., vol. 59, PWN, Warsaw, 1975.

4. J. P. R. Christensen, Theorems of Namioka and B. E. Johnson type for upper semi-continuous and compact-valued set-valued mappings, Proc. Amer. Math. Soc. 86 (1982), 649-655.

5. R. W. Hansell, A measurable selection and representation theorem in nonseparable spaces, Measure Theory (D. Kolzow and D. Maharam-Stone, eds.), Proceedings, Oberwolfach 1983, Lecture Notes in Math., vol. 1089, Springer-Verlag, New York and Berlin, 1984.

6. __ On Borel mappings and Baire functions, Trans. Amer. Math. Soc. 194 (1974), 195211.

7. R. W. Hansell, J. E. Jayne, and M. Talagrand, First class selectors for weakly upper semicontinuous multi-valued maps in Banach spaces, J. Reine Angew. Math. 361 (1985), 201220.

8. W. Hurewicz, Relativ perfekte Teile von Punktmengen und Mengen (A), Fund. Math. 12 (1928), 78-109.

9. J. E. Jayne and C. A. Rogers, Upper semi-continuous set-valued functions, Acta Math. 149 (1982), 87-125.

10. _ Borel selectors for upper semi-continuous multi-valued functions, J. Funct. Anal. 56 (1984), 279-299.

11. __ Borel selectors for upper semi-continuous set-valued mappings, Acta Math. 155 (1985), 41-79.

12. Sci. Paris Sér. I Math. 299 (1984), 125-128.

13. K. Kuratowski, Topology, Vol. 1, 5th ed., Academic Press, New York, 1966.

14. K. Kuratowski and C. Ryll-Nardzewski, A general theorem on selectors, Bull. Acad. Polon. Sci. Ser. Math. Phys. Astron. 13 (1965), 397-403. 
15. I. Labuda, Multi-valued Namioka theorems, Math. Scand. 58 (1986), 227-235.

16. I. Namioka, Separate continuity and joint continuity, Pacific J. Math. 51 (1974), 515-531.

17. J. Saint-Raymond, Jeux topologiques et espaces de Namioka, Proc. Amer. Math. Soc. 87 (1983), 499-504.

18. C. Stegall, Functions of the first Baire class with values in Banach spaces, Proc. Amer. Math. Soc. 111 (1991), 981-992.

19. R. W. Hansell, First class selectors for upper semi-continuous multifunctions, J. Funct. Anal. 75 (1987), 382-395.

Stat.-Math. Division, Indian Statistical Institute, 203 B. T. Road, Calcutta 700 035, INDIA 